\title{
Probing Half-odd Topological Number with Cold Atoms in a Non-Abelian Optical Lattice
}

\author{
Feng Mei ${ }^{1,3}$, Shi-Liang Zhu ${ }^{2}$, Xun-Li Feng ${ }^{1}$, Zhi-Ming Zhang ${ }^{1}$ 田 and C. H. Oh团 \\ ${ }^{1}$ Laboratory of Photonic Information Technology, LQIT \& SIPSE, \\ South China Normal University, Guangzhou 510006, China \\ ${ }^{2}$ Laboratory of Quantum Information Technology and SPTE, \\ South China Normal University, Guangzhou, China \\ ${ }^{3}$ Centre for Quantum Technologies and Department of Physics, \\ National University of Singapore, 3 Science Drive 2, Singapore 117543, Singapore
}

(Dated: June 15, 2018)

\begin{abstract}
We propose an experimental scheme to probe the contribution of a single Dirac cone to the Hall conductivity as half-odd topological number sequence. In our scheme, the quantum anomalous Hall effect as in graphene is simulated with cold atoms trapped in an optical lattice and subjected to a laser-induced non-Abelian gauge field. By tuning the laser intensity to change the gauge flux, the energies of the four Dirac points in the first Brillouin zone are shifted with each other and the contribution of the single Dirac cone to the total atomic Hall conductivity is manifested. We also show such manifestation can be experimentally probed with atomic density profile measurements.
\end{abstract}

PACS numbers: 37.10.Jk, 73.43.-f, 67.85.Lm, 71.10.Fd

\section{INTRODUCTION}

With easy production of graphene, this strictly twodimensional material has evoked strong interest in condense matter and high energy physics in the past few years, for instance, the integer quantum Hall effect (QHE) has been observed in graphene [1]. Remarkably, the transverse Hall conductivity has an anomalous form: $\sigma_{x y}=4(n+1 / 2) e^{2} / h(n=0, \pm 1, \ldots)$, where $n$ is the Landau level (LL) index and the factor 4 accounts for double valley and double spin degeneracy. Note that the conductance sequence is shifted with respect to the standard QHE sequence by $1 / 2$. This unusual sequence is now well understood as arising from the fact that the zero energy LL has half of the higher LLs degeneracy [2], and the Hall conductivity for each Dirac fermion is half-integer quantized as $(n+1 / 2) e^{2} / h,(n=0, \pm 1, \ldots)[3]$. Due to the presence of fermion doubling theorem [4], which states that for a time reversal invariant system Dirac points must come in pairs, the contribution of the single Dirac cone to the total Hall conductivity of the graphene is hidden. Even though one can find some lattice models which have an odd number of massless Dirac cones to avoid the fermion doubling theorem, the final Hall conductivity of the model is still integer quantized because of the hidden massive Dirac fermions [5]. In fact, the key reason lies in the famous Thouless-Kohmoto-Nightingale-den Nijs (TKNN) formula which guarantees that the total Hall conductivity is quantized as some integer numbers if the Fermi energy stays in the energy gap [6]. So far experiment is still lacking to demonstrate the half-integer Hall conductivity contributed from a single Dirac cone.

\footnotetext{
*Electronic address: zmzhang@scnu.edu.cn
}

†Electronic address: phyohch@nus.edu.sg
Very recently, Watanabe and colleagues proposed a model to show that each Dirac cone does indeed contribute to the Hall conductivity with half-odd topological numbers [7]. In their work, they constructed a lattice model in graphene with complex second nearest-neighbor hopping integral among some particular lattice sites. The most interesting feature of the model is that the energies of the two Dirac points are shifted with each other, but their massless Dirac cones are still preserved. Through computing the topological number for the shifted Dirac cones, the half-integer contribution to the Hall conductivity from single Dirac cone can be demonstrated. However, as the authors themselves pointed out in their work, the lattice model is fictitious and it is extremely hard to realize the unusual second nearest-neighbor hopping proposed there.

On the other hand, optical lattices populated with cold atoms offer a very promising alternative avenue to explore rich fundamental phenomena of condensed matter physics [8]. For example, ultracold atoms in the optical lattices can be used to simulate the Bose-Hubbard model and the fractional quantum Hall effect, etc. 9]. In analogy to the graphene, it is also proposed that the Dirac fermions can be simulated and detected by the ultracold atoms in the honeycomb optical lattice [10] and then demonstrated in details that the massive and massless Dirac particles can be realized in realistic conditions [11]. In addition to the honeycomb lattices, the Dirac fermions can also be generated from a line-centered-square lattice or a lattice with $T_{3}$ symmetry [12]. Furthermore, the Dirac cones can be realized with a square lattice subjected to an artificial non-Abelian gauge potential. The artificial gauge potentials acting on neutral atoms have recently attracted considerable interest [13]. The gauge potential can lead to an effective magnetic field and provide opportunities to simulate the physics of charges subjected to magnetic fields. Many recent works have shown 


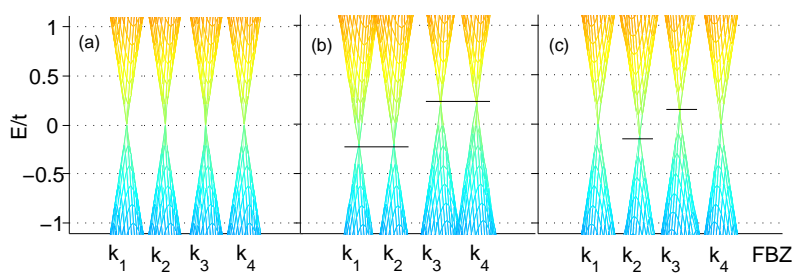

FIG. 1: (Color online) The dispersion relation near the four Dirac cones in the FBZ for the different parameters $\alpha$ and $\beta$. (a) $\alpha=\beta=\pi / 2$; (b) $\alpha=\pi / 2+0.1, \beta=\pi / 2$; (c) $\alpha=\pi / 2+0.05, \beta=\pi / 2-0.05$.

that the Abelian and non-Abelian gauge fields can be realized using optical dressing [14] or laser-assisted hopping [15] in optical lattice for observing the Hofstadter butterfly [16], atomic QHE [17 19], topological insulators 20], axion electrodynamics 21] and Majorana fermion [22]. Both Abelian and non-Abelian gauge fields in cold atoms have recently been generated experimentally [23].

Motivated by these ongoing developments, we propose an experimental scheme to manipulate single Dirac cone and probe its contribution to Hall conductivity as halfodd topological numbers with cold atoms in the optical lattices subjected to an artificial non-Abelian gauge potential. We devise a distinct method to engineer the Dirac cone through changing the non-Abelian gauge flux in optical lattice. In the $\pi$-flux regime, there will be four zero energy Dirac points in the first Brillouin zone which leads to the anomalous Hall effect as in graphene. The key point in our paper is that, through changing the non-Abelian gauge flux, the energies of the four Dirac points are shifted with each other, but their positions and massless Dirac cone characters remain intact. By manipulating the Dirac cones and calculating the corresponding topological Chern numbers, the contribution of the single Dirac cone to the total atomic Hall conductivity as half-odd topological numbers is demonstrate indirectly. In our model, different gauge fluxes can be easily adjusted through tuning the laser field intensity. Furthermore, we show that the atomic Hall conductivity could be detected with the standard density profile measurement used in cold atomic systems.

\section{SUMULATING AND ENGINEERING DIRAC CONE}

Let us consider a two-component ultracold fermion gas trapped in a two dimensional square optical lattice with the lattice sites $(x=m a, y=n a)$, where $a$ is the lattice spacing, $t$ is the nearest-neighbor hopping strength and the integers $m$ and $n$ are the position of the lattice site. We assume that the atomic collisions are rendered negligible through adjusting Feshbach resonance [24]. The optical lattice setup is subjected to a gauge potential as $\vec{A}=\left(\alpha \sigma_{y}, \beta \sigma_{x}+2 \pi \Phi m\right)$, where $\sigma_{x(y)}$ is the Pauli operator of the two atomic components labelled by two atomic sublevels of a hyperfine manifold, $\Phi$ is the uniform Abelian magnetic flux per unit cell and we set $c=\hbar=e=a=1$. This gauge potential may be generated using a two dimensional optical superlattice based on laser-assisted spin-dependent hopping 18, 19, 21, 25]. The gauge fluxes $(\alpha, \beta)$ are determined by the Rabi frequencies of the two-photon off-resonant transition and can be tuned via changing the laser field intensity [18, 19, 21, 25]. The tight-binding Hamiltonian of the system is written as

$$
H=-t \sum_{m, n}\left(c_{m+1, n}^{\dagger} e^{i \alpha \sigma_{y}} c_{m, n}+c_{m, n+1}^{\dagger} e^{i\left(\beta \sigma_{x}+2 \pi \Phi m\right)} c_{m, n}\right)+H . c
$$

where $c_{m, n}$ is a 2-component field operator defined on a lattice site $(m, n)$. We firstly study the system in the non-Abelian gauge potential when the uniform Abelian gauge flux $\Phi=0$. By diagonalizing the above Hamiltonian, one can get the single-particle energy spectra as $E_{k}=h_{0}(k) \pm \sqrt{h_{x}^{2}(k)+h_{y}^{2}(k)}$, where $h_{0}(k)=-2 t\left(\cos (\alpha) \cos \left(k_{x}\right)+\cos (\beta) \cos \left(k_{y}\right)\right), h_{x}(k)=$ $-2 t \sin (\beta) \sin \left(k_{y}\right)$ and $h_{y}(k)=-2 t \sin (\alpha) \sin \left(k_{x}\right)$. When the gauge fluxes $\alpha=\beta=\pi / 2$ ( $\pi$-flux regime), this dispersion relation leads to four Dirac points around which the dispersion relation is linear [19]. The four Dirac points are located at $\mathbf{k}_{1}=(0,0), \mathbf{k}_{2}=(0, \pi), \mathbf{k}_{3}=(\pi, 0)$, $\mathbf{k}_{4}=(\pi, \pi)$ in the first Brillouin zone (FBZ) with zero energies. Note that this $\pi$-flux gauge potential is in the
Abelian regime because its Wilson loop $|W|=2$ [18]. Interestingly, the relative energies of the four Dirac points could be shifted through changing the gauge flux from the Abelian to the non-Abelian regime $(|W|<2)$. This can be seen clearly by expanding the Hamiltonian in Eq.(1) around the four Dirac points. Apart the conventional linear dispersion relation related to the Dirac Hamiltonian, we find that the energies of Dirac points become

$$
\begin{aligned}
& \delta E_{1(4)}= \pm 2 t(\cos (\alpha)+\cos (\beta)), \\
& \delta E_{2(3)}= \pm 2 t(\cos (\alpha)-\cos (\beta)) .
\end{aligned}
$$

In the case of Abelian $\pi$-flux regime $(\alpha=\beta=\pi / 2)$, the energies of the four Dirac points are zero, which agrees with the previous description. However, if the 
gauge fluxes have been changed into the non-Abelian regime $\alpha=\pi / 2+\triangle \alpha$ and $\beta=\pi / 2+\triangle \beta$, their energies are $\delta E_{1(4)}=\mp 2 t(\sin (\triangle \alpha)+\sin (\triangle \beta))$ and $\delta E_{2(3)}=$ $\mp 2 t(\sin (\triangle \alpha)-\sin (\triangle \beta))$ and would be shifted with respect to each other. As shown in Fig. 1, the energies of the four Dirac points vary with the gauge fluxes but the massless Dirac cone character is still kept. In particular, we shift the energies of four and two Dirac points respectively in Fig.1(b) and (c).

\section{HALF-ODD TOPOLOGICAL NUMBER}

Now we turn to address the Hall conductivity in the Dirac cone regime. According to the famous TKNN expression [6], the quantized value of the total Hall conductivity is described as

$$
\sigma_{x y}=\frac{1}{(2 \pi)^{2} i} \sum_{E_{\lambda}<E_{F}} \int_{T_{M B Z}^{2}} \sum_{\lambda}\left(\left\langle\frac{\partial u_{\lambda}}{\partial k_{x}}|| \frac{\partial u_{\lambda}}{\partial k_{y}}\right\rangle-\left\langle\frac{\partial u_{\lambda}}{\partial k_{y}}|| \frac{\partial u_{\lambda}}{\partial k_{x}}\right\rangle\right) d \mathbf{k}
$$

where $\left|u_{\lambda}\right\rangle$ is the wave function of the energy band $\lambda$ and $T_{M B Z}^{2}$ is the magnetic Brillouin zone (MBZ). The wave function $\left|u_{\lambda}\right\rangle$ forms a U(1) fibre bundle on the MBZ and the corresponding quantized value of the Hall conductivity is the first Chern number which is a topological invariant of the U(1) bundle. If the Fermi energy $E_{F}$ lies in one of the energy gaps, the total quantized value of the Hall conductivity is a sum of the first Chern numbers corresponding to each energy band below the Fermi energy. In Fig. 2, using lattice gauge theory [26], we calculate numerically the Hall conductivity in the relativistic regime in the condition of Fig.1 (same indexing). Here the laser-induced uniform abelian gauge flux plays the role of external magnetic field, which is used to break the time-reversal symmetry for getting the quantum Hall conductivity. In the presence of this magnetic field, the energy spectrum of the Dirac cones are splitted into many relativistic LLs. We also plot the relativistic LLs in each Dirac cone and the corresponding half-odd topological number sequences in the lower part of Fig. 2.

In Fig. 2(a), the calculated result is for the original lattice model without shifting the Dirac cones. The Hall conductivity associated to each energy gap in the Dirac cone regime is $\{-10,-6,-2,2,6,10\}$, which has a step of 4 as that in graphene. Compared with the usual Hall conductivity, it has an anomalous form $\sigma_{x y}=4(n+1 / 2) / h$ $(n=0, \pm 1, .$.$) . This anomaly arises from the fact that$ each Dirac cone contributes to the total Hall conductivity as a half-odd topological number sequence: $n+1 / 2$. The factor 4 accounts for double valley and double spin degeneracy. As indicated in Fig. 2(a), the sum of the half-odd topological numbers over the four Dirac cones in each gap just corresponds to the calculated Chern number. However, because the TKNN formula guarantees that the quantized values of the Hall conductivity for each gap are integer topological Chern numbers [6], the contribution of a single Dirac cone to the Hall conductivity as half-odd topological numbers has been hidden.
To manifest the contribution of the single Dirac cone, in Fig. 2(b-c), we shift the energies of the Dirac cones and expect that the effect of this shift on the Hall conductivity can give evidence of their own contribution. In Fig. 2(b), the Hall conductivity is calculated when one pair of Dirac cones is shifted equally toward positive energy and the other pair toward negative energy, and the relativistic LLs in each shifted Dirac cone are also plotted in the lower part of the figure. The calculated result shows that the new Hall sequence is $\{-10,-8,-6,-4,-2,0,2,4,6,8,10\}$. By comparison, one also can find that the positions of the shifted relativistic LLs coincide with the positions of the jumps of the new Hall plateaus, i.e. each Hall plateau corresponds to one of the new energy gaps induced by the above shift. This means that the appearance of the new Hall sequence is just because of the shifted Dirac cones. Furthermore, when the Fermi energy lies in one of the new gaps, as indicated in the lower part of Fig. 2(b), each shifted Dirac cone contributes to the total Hall conductivity of the gap with a half-odd topological number. It is found that the sum of the four half-odd topological numbers corresponding to each gap is equal to the numerical calculated Chern number of the gap. For example, for the central gap, the two positive (negative) shifted Dirac cones contribute equally to the Hall conductivity of the gap with a half-odd topological number $-1 / 2(1 / 2)$, their sum $2 \times(-1 / 2+1 / 2)$ is just equal to the numerical calculated Chern number 0 . As seen from this agreement, the half-odd contribution of single Dirac cone is manifested. In Fig. 2(c), we only shift one Dirac cone toward positive and one toward negative energy, and the other two ones keep intact. The induced new Hall sequence $\{-10,-9,-7,-6,-5,-3,-2,-1,1,2,3,5,6,7,9,10\}$ becomes more complicated. However, one can still find that the sum of the half-odd topological number sequences in the Dirac cones with and without shift agrees with the calculated Chern number sequence. In fact, different shifting cases make no difference on the underlying agreement. Note that this agreement is very surprising, 

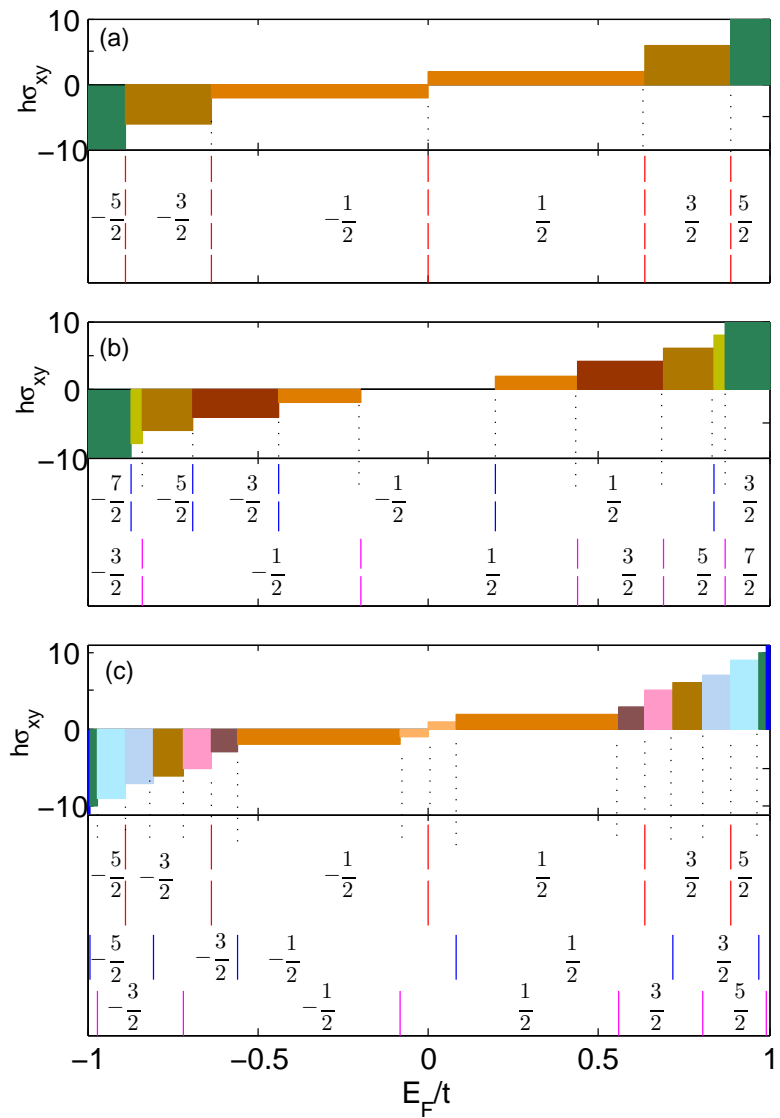

FIG. 2: (Color online) In the conditions of Fig.1 (same indexing), the Hall plateaus against the Fermi energy in the relativistic regime is shown when $\Phi=1 / 121$. For comparison, in the lower part of each figure, we also plot the relativistic LLs and show the half-odd Chern number corresponding to each gap in the four Dirac cones respectively. Here we choose low magnetic flux $(\Phi=1 / 121)$ so that we can get more LLs in the Dirac cone for rendering their contribution to the Hall conductivity more pronounced.

because there is no obvious reason to show that the superposition of field theory for the shifted Dirac cones can also give the same Chern numbers 7]. So, based on these agreements, we can demonstrate indirectly the contribution of single Dirac cone as half-odd topological numbers. Due to TKNN, one can only have indirect signatures of half-odds, but our setup allows one to have such partial signs in a clearer and simpler fashion as compared to existing one [7].

\section{DETECTION}

In the following, we explain how to detect the atomic Hall conductivity with the density profile measurement [27, 28]. The density profile of the trapped atoms can be measured through the time-of-flight (TOF) imaging with the light absorption [29] instead of momentum distribution, which is specific to two dimensional system [30].
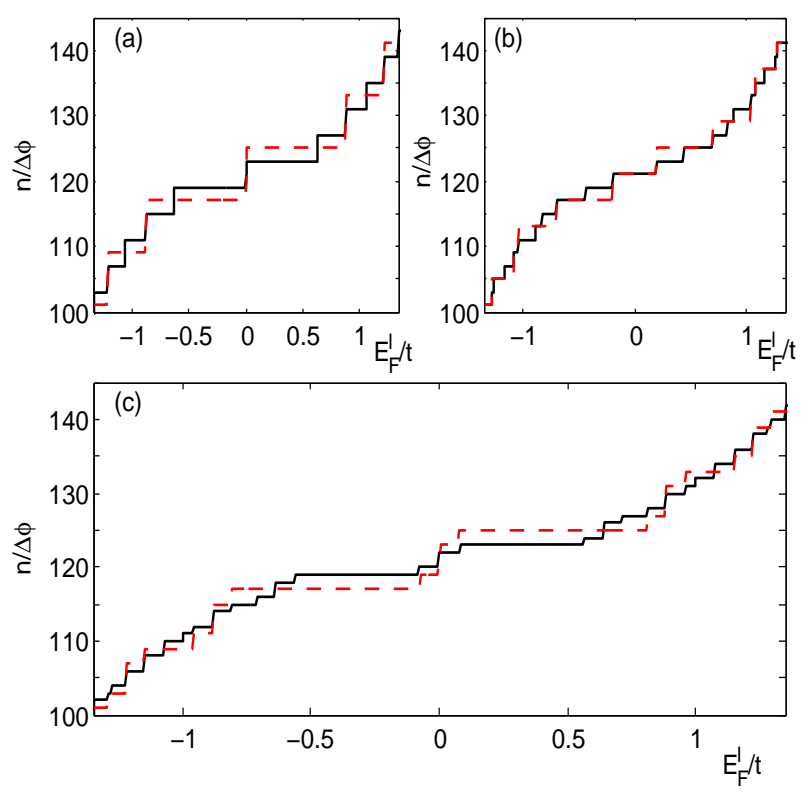

FIG. 3: (Color online) The atomic density profiles $n\left(E_{F}^{l}\right) / \triangle \Phi$ against the local Fermi energy for the Dirac Fermions in the condition of Fig. 1 (same indexing) and the solid (dash) line corresponding to $\Phi=1 / 121(2 / 121)$.

Note that usually an external harmonic trap has been applied to confine the atoms in the optical lattice. If the trap potential varies slowly compared to the lattice spacing, the local density approximation (LDA) is well satisfied [31]. Under this approximation, the local Fermi energy is defined as $E_{F}^{l}(r)=E_{F}-V(r)$, where $E_{F}$ is the Fermi energy in the trap center and $V(r)=m \omega^{2} r^{2} / 2$ is the global harmonic trap potential. The atomic density at temperature $T$ is written as

$$
n\left(E_{F}^{l}\right)=\int \frac{d \mathbf{k}}{(2 \pi)^{2}} f\left(k, E_{F}^{l}\right)
$$

where $f\left(k, E_{F}^{l}\right)=1 /\left\{\exp \left[\left(E_{k}-E_{F}^{l}\right) / T\right]+1\right\}$ is the FermiDirac distribution. If the local Fermi energy $E_{F}^{l}(r)$ falls in a energy gap as plotted in Fig. 2, the atomic density $n\left(E_{F}^{l}\right)$ will depict a plateau. The Hall conductivity is related to the atomic density according to the Streda formula $\sigma_{x y}=\left.\frac{1}{h} \frac{\partial n}{\partial \Phi}\right|_{E_{F}^{l}, T}$. As can be seen from this formula, to measure the Hall conductivity, we need to choose two magnetic flux values, and identify the plateaus in both density profiles that correspond to the same gap. The density difference of the two plateaus divided by the magnetic flux difference gives the Hall conductance of the gap. In Fig. 3, at low temperature, we plot the density profiles of the atomic gas in the relativistic regime in the condition of Fig. 1 (same indexing). Each case contains two density profiles for $\Phi_{1}=1 / 121$ and $\Phi_{2}=2 / 121$ and $\triangle \Phi=\Phi_{2}-\Phi_{1}=1 / 121$. Through comparing the two density profiles for $\Phi_{1}$ and $\Phi_{2}$, the atomic Hall conductivity can be derived as $h \sigma_{x y}=\left(n_{2}-n_{1}\right) / \triangle \Phi$ [27, 28]. 
One can find that the derived atomic Hall conductivity from the calculated theoretical result in Fig. 3 agrees with the prediction in Fig. 2 .

\section{DISCUSSION AND SUMMARY}

Finally, we give a brief discussion on the density profile measurement from an experimental point of view. Due to the external harmonic trap, the local Fermi energy decreases continuously from the trap center to edge. If we let the Fermi energy at the center of the trap higher than $t$, it automatically scans from $t$ to $-t$. The local Fermi energy at the center of the trap can be increased by loading more atoms in the trap 32. It is not difficult to make the local Fermi energy higher than $t$, as it has been done in many previous experiment of fermions in optical lattices [32]. Moreover, there is no need to look for the exact value of the local Fermi energy as long as we do the comparison between plateau densities in the same energy gap. It means that, to a certain degree, the measurement result is insensitive to the influence of the experimental noise on the parameters $(\Phi, \alpha, \beta)$, which also reflects the topological character of the quantized value of the Hall conductivity. Note that the measure result is also influenced by the temperature and the external trap frequency. The temperature is related to the visibility of plateaus in density profile. As shown in [27], the temper- ature of the atoms should be cooled to the order of nk for observing discernible plateaus, which is easy than realizing the degenerate Fermi gases. The external harmonic trap frequency determines the validity of the LDA. If the trap potential varies smoothly so that the confinement scale does not approach the scale of lattice constant, the LDA will be safe. Obviously, this requirement is within the current experimental technology.

In summary, with cold atoms in a non-Abelian optical lattice, we have proposed an experimental scheme to simulate the quantum anomalous Hall effect as in graphene. By tuning the laser intensity to change the gauge fluxes, we show that the contribution of a single Dirac cone to the Hall conductivity is a half-odd topological number sequence, and such topological numbers are detectable through the standard density profile measurement used in cold atom systems.

F. Mei thanks N. Goldman, Hui Zhai and R.O. Umucalılar for helpful discussions. This work was supported by the NSFC under Grant No. 60978009, No. 10974059 and No. 11074079, the SKPBR of China (No.2007CB925204, No.2009CB929604 and No.2011CB922104), and NUS Academic Research (Grant No. WBS: R-710-000-008-271).
[1] K.S. Novoselov et al., Nature (London) 438, 197 (2005); Y. Zhang et al., ibid. 438, 201 (2005).

[2] V.P. Gusynin and S.G. Sharapov, Phys. Rev. Lett. 95, 146801 (2005).

[3] M.Z. Hasan, Physics 3, 62 (2010).

[4] L-H. Karsten and J. Smit, Nuc. Phys. B 183, 103 (1981); H.B. Nielsen and M. Ninomiya, ibid 185, 20 (1981); 193, 173 (1981).

[5] Y. Hatsugai et al., Phys. Rev. B 54, 4898 (1996).

[6] D. J. Thouless et al., Phys. Rev. Lett. 49, 405 (1982).

[7] H. Watanabe, Y. Hatsugai, and H. Aoki, Phys. Rev. B 82, 241403(R) (2010).

[8] I. Bloch, J. Dalibard, and W. Zwerger, Rev. Mod. Phys. 80, 885 (2008).

[9] D. Jaksch et al., Phys. Rev. Lett. 81, 3108 (1998); M. Greiner et al., Nature (London) 415, 39 (2002); M. A. Baranov, K. Osterloh, and M. Lewenstein, Phys. Rev. Lett. 94, 070404 (2005).

[10] S.-L. Zhu et al., Phys. Rev. Lett. 98, 260402 (2007).

[11] K. L. Lee et al., Phys. Rev. A 80, 043411 (2009).

[12] R. Shen et al.,Phys. Rev. B 81, 041410 (2010); D. Bercioux et al.,Phys. Rev. A 80, 063603 (2009).

[13] J. Dalibard et al., arXiv:1008.5378

[14] J. Ruseckas et al.,, Phys. Rev. Lett. 95, 010404 (2005).

[15] D. Jaksch and P. Zoller, New J. Phys. 5, 56 (2003).

[16] D. Hofstadter, Phys. Rev. B 14, 2239 (1976).

[17] S.-L. Zhu et al., Phys. Rev. Lett. 97, 240401 (2006); X.J. Liu et al., ibid., 98, 026602 (2007); Phys. Rev. A 81, 033622 (2010); Y. Li, C. Bruder, and C. P. Sun, Phys.
Rev. Lett. 99, 130403 (2007).

[18] N. Goldman et al., Phys. Rev. A 79, 023624 (2009).

[19] J. M. Hou, W. X. Yang, and X. J. Liu, Phys. Rev. A 79, 043621 (2009); N. Goldman et al., Phys. Rev. Lett. 103, 035301 (2009).

[20] N. Goldman et al., Phys. Rev. Lett. 105, 255302 (2010); Phys. Rev. A 83, 063601 (2011).

[21] A. Bermudez et al., Phys. Rev. Lett. 105, 190404 (2010).

[22] S.-L. Zhu et al., Phys. Rev. Lett. 106, 100404 (2011).

[23] Y. J. Lin et al., Nature (London) 462, 628 (2009); ibid. 471, 83 (2011).

[24] J. Billy et al., Nature (London) 453, 891 (2008); R. Giacomo et al., ibid. 453, 895 (2008).

[25] Z. Lan et al., arXiv:1102.5283.

[26] Y. Hatsugai, J. Phys. Soc. Jpn. 73, 2604 (2004); T. Fukui et al., ibid. 74, 1673 (2005).

[27] R.O. Umucalılar et al., Phys. Rev. Lett. 100, 070402 (2008);

[28] L.B. Shao et al., Phys. Rev. Lett. 101, 246801 (2008); A Bermudez et al., New J. Phys. 12, 033041 (2010).

[29] M.W. Zwierlein et al., Science 311, 492 (2006); G.B. Partridge et al., ibid. 311, 503 (2006); C.-L Hung et al., Nature (London) 470, 236 (2011).

[30] Zoran Hadzibabic et al., Nature (London) 441, 1118 (2006); N. Read and N.R. Cooper, Phys. Rev. A 68, 035601 (2003).

[31] W. Yi and L.-M. Duan, Phys. Rev. A 74, 013610 (2006).

[32] T. Esslinger, Annu. Rev. Condens. Matter Phys. 1, 129 (2010). 